\title{
Focal irregularities in 7-Tesla MRI of unruptured intracranial aneurysms as an indicator for areas of altered blood-flow parameters
}

\author{
Matthias Millesi, MD, ${ }^{1,3}$ Engelbert Knosp, MD, ${ }^{1}$ Georg Mach, MSc, ${ }^{3}$ Johannes A. Hainfellner, MD, ${ }^{4}$ \\ Gerda Ricken, MSc, ${ }^{4}$ Siegfried Trattnig, MD, ${ }^{5}$ and Andreas Gruber, MD ${ }^{1-3}$ \\ ${ }^{1}$ Department of Neurosurgery, ${ }^{3}$ Cerebrovascular Research Group Vienna, ${ }^{4}$ Institute of Neurology, and 5 High Field MR Centre, \\ Department of Biomedical Imaging and Image-Guided Therapy, Medical University of Vienna; and 'Department of Neurosurgery, \\ Johannes Kepler University Linz, Austria
}

\begin{abstract}
OBJECTIVE In the last several decades, various factors have been studied for a better evaluation of the risk of rupture in incidentally discovered intracranial aneurysms (IAs). With advanced MRI, attempts were made to delineate the wall of IAs to identify weak areas prone to rupture. However, the field strength of the MRI investigations was insufficient for reasonable image resolution in many of these studies. Therefore, the aim of this study was to analyze findings of IAs in ultra-high field MRI at 7 Tesla (7 T).

METHODS Patients with incidentally found IAs of at least $5 \mathrm{~mm}$ in diameter were included in this study and underwent $\mathrm{MRI}$ investigations at $7 \mathrm{~T}$. At this field strength a hyperintense intravascular signal can be observed on nonenhanced images with a brighter "rim effect" along the vessel wall. Properties of this rim effect were evaluated and compared with computational fluid dynamics (CFD) analyses.
\end{abstract}

RESULTS Overall, 23 aneurysms showed sufficient image quality for further evaluation. In 22 aneurysms focal irregularities were identified within this rim effect. Areas of such irregularities showed significantly higher values in wall shear stress and vorticity compared to areas with a clearly visible rim effect ( $p=0.043$ in both).

CONCLUSIONS A hyperintense rim effect along the vessel wall was observed in most cases. Focal irregularities within this rim effect showed higher values of the mean wall shear stress and vorticity when compared by CFD analyses.

Therefore, these findings indicate alterations in blood flow in IAs within these areas.

https://thejns.org/doi/abs/10.3171/2019.9.FOCUS19489

KEYWORDS intracranial aneurysm; 7 Tesla; rim effect; computational fluid dynamics; CFD analyses

I NTRACRANIAL aneurysms (IAs) show a prevalence of approximately $3 \%$ in the general population, and due to the increased availability of neuroradiological imaging, an increasing number of IAs are discovered incidentally. ${ }^{7,35}$ Previous studies analyzing the natural course of IAs have identified several factors such as larger aneurysm size and location to be important for the annual risk of aneurysm rupture., $90,36,37$ Therefore, indications for treatment of incidentally found IAs are still mainly based on the maximum diameter. ${ }^{28}$ However, because smaller aneurysms constitute up to half of all aneurysms presenting with subarachnoid hemorrhage (SAH), further attempts have been made to better estimate the risk of aneurysm rupture. ${ }^{12,28}$ Because IAs mainly occur at or close to vessel bifurcations with presumed intraaneurysmal alterations in blood flow, studies have focused on intraluminal flow parameters. ${ }^{2,20}$ In this sense, based on computational fluid dynamics (CFD) analyses, distinct intraaneurysmal flow patterns and changes in blood flow parameters such as wall shear stress were found at sites associated with aneurysm initiation, growth, rupture, or bleb formation along the aneurysm dome. . $^{2,3,5,19,25}$

Additionally, in the last decade, attempts were made to study the intracranial vessel wall and associated pathologies by noninvasive neuroradiological imaging at different values of the magnetic field strength..$^{1,23,29,30}$ In a small

ABBREVIATIONS CFD = computational fluid dynamics; $D S A=$ digital subtraction angiography; $I A=$ intracranial aneurysm; ICA = internal carotid artery; $M C A=$ middle cerebral artery; MPRAGE = magnetization-prepared rapid acquisition gradient echo; $\mathrm{SAH}=$ subarachnoid hemorrhage; TSE $=$ turbo-spin echo; $7 \mathrm{~T}=7 \mathrm{Tesla}$. SUBMITTED June 25, 2019. ACCEPTED September 9, 2019. INCLUDE WHEN CITING DOI: 10.3171/2019.9.FOCUS19489. 
number of studies the characteristics of ultra-high field MRI at $7 \mathrm{~T}$ have also been investigated in first attempts at identification of IAs or their vessel wall. ${ }^{11,14,32-34,38,39,41} \mathrm{In}$ these studies, a hyperintense intraluminal signal in nonenhanced images was described. ${ }^{14,32,41}$ However, a dedicated investigation of this finding and comparison with alterations in blood flow parameters has not been performed thus far. Hence, the aim of our study was to evaluate this hyperintense signal on nonenhanced ultra-high field MRI in a prospective patient series with unruptured IAs in combination with CFD analyses.

\section{Methods}

In this study, patients harboring incidentally discovered IAs between 2009 and 2016 were included. Patients needed to have at least 1 unruptured IA with a diameter of at least $5 \mathrm{~mm}$. The cutoff of $5 \mathrm{~mm}$ was chosen to test the findings on 7-T ultra-high field MRI in larger aneurysms in a first step for investigation of the feasibility of this protocol. Fusiform aneurysms and patients who presented with ruptured aneurysms, underwent prior treatment for another IA, or had contraindications to ultrahigh field MRI were excluded from this study. The study was approved by the ethics committee of the Medical University of Vienna and all patients provided informed consent.

\section{Routine Neuroimaging}

Each patient underwent routine preoperative imaging. This imaging consisted of CT (including CT angiography), MRI (including MR angiography), and invasive digital subtraction angiography (DSA) for further treatment planning. In each IA, the exact location, maximum diameter, and its position on the parent artery (bifurcation or sidewall) were noted.

\section{Ultra-High Field MRI}

In addition to routine neuroimaging, all patients underwent ultra-high field MRI that was performed in a wholebody 7-T MRI unit (Siemens Healthcare) with a gradient strength of $40 \mathrm{mT} / \mathrm{m}$ using a 32-channel transmit/receive coil. The MRI sequence performed for this study was a nonenhanced magnetization-prepared rapid acquisition gradient echo (MPRAGE) sequence (TR/TE 3850/3.84 msec, isotropic voxel size $0.5 \mathrm{~mm}^{3}$ ).

On nonenhanced MPRAGE images, besides the hyperintense intraluminal signal of the intracranial vasculature, the presence of a rim enhancement adjacent to the inner wall of IAs was noted..$^{14,32,41}$ In this study, we termed this bright signal the "rim effect" and analyzed this characteristic for its existence in all scanned aneurysms, and if present, for its signal quality. Furthermore, if this rim effect was noted, it was evaluated for its completeness or potential focal interruptions. The primary orientation for image analyses was axial slices, however, to reduce the misinterpretation of potential artifacts such as a focal interruption, the existence of such a finding was always evaluated in multiple slices together with coronal and sagittal orientation. Additionally, a relationship between such a potential interruption of the rim effect and possible small
TABLE 1. Imaging parameters

\begin{tabular}{lcc}
\hline \multirow{2}{*}{ Parameter } & \multicolumn{2}{c}{ Sequences \& Protocol Used for 7-T MRI } \\
\cline { 2 - 3 } & T1 MPRAGE & T2 TSE 2D \\
\hline Voxel dimensions $\left(\mathrm{mm}^{3}\right)$ & $0.5 \times 0.5$ & $0.4 \times 0.4$ \\
\hline Matrix size & $448 \times 403$ & $528 \times 528$ \\
\hline No. of slices & 256 & 34 \\
\hline FOV $\left(\mathrm{mm}^{2}\right)$ & $230 \times 172$ & $230 \times 172$ \\
\hline Slice thickness $(\mathrm{mm})$ & 0.5 & 3 \\
\hline TE $(\mathrm{msec})$ & 3.5 & 69 \\
\hline TI (msec) & 1700 & \\
\hline TR (msec) & 3850 & 7000 \\
\hline TA (mins:sec) & $12: 17$ & $03: 39$ \\
\hline GRAPPA factor & 2 & 2 \\
\hline Bandwidth $(\mathrm{Hz} /$ pixel) & 180 & 287 \\
\hline Flip angle $\left({ }^{\circ}\right)$ & 9 & 146 \\
\hline
\end{tabular}

FOV = field of view; GRAPPA = generalized autocalibrating partially parallel acquisitions.

blood vessels leaving the aneurysms or the parent vessels was also evaluated.

In addition to the study MRI sequence, standard T2 turbo-spin echo (TSE) images for morphological reference images were acquired. The complete scanning protocol lasted approximately 30 minutes. For details of the protocol, see Table 1.

\section{CFD Analyses}

Nonenhanced T1-weighted MPRAGE images acquired by the 7-T MRI scanner were processed for CFD calculations. ${ }^{13}$ Surface 3D models were created with open-source software ITK-SNAP, together with MeshLab for smoothing of surface models. ${ }^{6,40}$ In a next step, all models were further processed using NetGen for creation of triangular elements covering the complete aneurysm surface together with inflow and outflow blood vessels. ${ }^{26}$

After formation of these 3D models, all data were transferred to COMSOL Multiphysics (COMSOL Inc.) and the governing 3D incompressible Navier-Stokes equations were solved in the whole model area for CFD analyses. Blood was assumed as an incompressible Newtonian fluid with a density of $1055 \mathrm{~kg} / \mathrm{m}^{3}$ and a blood viscosity of $0.0049 \mathrm{~Pa}-\mathrm{sec}^{22}$ The vessel walls were modeled rigid with no-slip boundary conditions. At the inlets a laminar, steady blood flow was assumed based on existing data on various intracranial vessels from the literature; at the outlets no pressure resistance was assumed. ${ }^{21}$

The evaluation of the calculated absolute value of wall shear stress vector $(\mathrm{Pa})$, vorticity $(1 / \mathrm{sec})$, and wall-directed force $(\mathrm{Pa})$ was performed and displayed in color maps at the slice where the rim effect showed the strongest signal. The wall-directed force is the component of the total stress that is perpendicular to the vessel wall, whereas the wall shear stress is equal to the tangential components. These calculated values of wall-directed force, vorticity, and wall shear stress were then compared between segments with a missing rim and the next segments with a clearly visible rim effect in the same aneurysms. 
TABLE 2. Patient characteristics

\begin{tabular}{lc}
\hline \multicolumn{1}{c}{ Characteristic } & Value \\
\hline No. of patients & 18 \\
\hline No. of IAs & 24 \\
\hline Female/male ratio & $5: 1$ \\
\hline Mean age (range), yrs & $55(42-74)$ \\
\hline Mean diameter (range), $\mathrm{mm}$ & $14.4(5.0-94.2)$ \\
\hline Aneurysm location, $\mathrm{n}(\%)$ & \\
\hline MCA & $8(33)$ \\
\hline ICA & $8(33)$ \\
\hline ACoA & $4(17)$ \\
\hline BA & $2(8)$ \\
\hline ACA & $1(4)$ \\
\hline PCA & $1(4)$ \\
\hline Aneurysm position, $\mathrm{n}(\%)$ & $20(83)$ \\
\hline Bifurcation & $4(17)$ \\
\hline Sidewall
\end{tabular}

$\mathrm{ACA}=$ anterior cerebral artery; $\mathrm{ACo} \mathrm{A}=$ anterior communicating artery; $\mathrm{BA}=$ basilar artery; $\mathrm{PCA}=$ posterior cerebral artery.

\section{Statistical Analysis}

For statistical analysis, we used SPSS statistical software (version 25.0, IBM Corp.). Categorical variables are shown as values and percentages, and continuous variables as means \pm standard deviations. Continuous variables of velocity, wall-directed force, vorticity, and wall shear stress were tested for normalcy using a KolmogorovSmirnov test. In case of normalcy, these values were compared using a Student t-test grouped together if the rim effect was visible or interrupted. A 2-sided $p$ value $<0.05$ was considered statistically significant.

\section{Results}

Overall, 30 patients with a total number of 45 unruptured intracranial saccular aneurysms were enrolled in the study. Of all patients, 21 harbored a single IA. More than 1 IA was present in 9 patients ( 2 aneurysms were present in 6 patients, and 3,4, or 5 aneurysms were present in 1 patient each). Of these 45 IAs, $36(80 \%)$ showed a diameter of at least $5 \mathrm{~mm}$ and underwent 7-T MRI in addition to routine preoperative neuroimaging.

The mean patient age was 55 years (range $42-74$ years) and the female to male ratio was 5:1. The most common symptoms that led to the diagnosis of an IA were headache without any signs of SAH in 7 patients (24\%) and incidental finding in patients with ischemic strokes in 5 patients (17\%), followed by vertigo in 5 patients (17\%).

\section{Ultra-High Field MRI at $7 \mathrm{~T}$}

The acquisition of 7-T MR images (T1-weighted MPRAGE images as well as T2-weighted TSE images) was feasible in all patients. Regarding the study sequence (nonenhanced MPRAGE T1-weighted sequences), image quality for further analysis was sufficient in 27 aneurysms (74\%) whereas distortion and insufficient quality were not-
TABLE 3. Evaluation of the rim effect

\begin{tabular}{cc}
\hline Variable & No. $(\%)$ \\
\hline Included IAs for evaluation & 24 \\
\hline Rim effect visible & $23(96)$ \\
\hline Rim effect not visible & $1(4)$ \\
\hline Signal intensity & 23 \\
\hline Thin & $17(74)$ \\
\hline Medium & $6(26)$ \\
\hline Completeness of rim effect & 23 \\
\hline Incomplete & $22(96)$ \\
\hline Complete & $1(4)$ \\
\hline Association to small branches & 22 \\
\hline No side branches & $18(82)$ \\
\hline Exiting side branches & $4(18)$ \\
\hline
\end{tabular}

ed in the remaining 9 aneurysms (26\%). Therefore, these cases were excluded from evaluation of the rim effect. In further imaging analyses 3 cases were found to be fusiform aneurysms and therefore were also excluded from this study. A complete list of all included aneurysms is given in Table 2.

\section{Aneurysm Locations and Characteristics}

Of the 24 aneurysms included in this study, the most common aneurysm location was along the middle cerebral artery (MCA; $\mathrm{n}=8$ aneurysms, 33\%) and internal carotid artery (ICA; $\mathrm{n}=8$ aneurysms, 33\%). The mean diameter of all aneurysms was $14.4 \mathrm{~mm}$ (range 5.0-94.2 mm). Based on their position along the parent artery, 20 aneurysms $(83 \%)$ were located at a vessel bifurcation whereas 4 were sidewall aneurysms (17\%). A complete list of aneurysm details is given in Table 2.

\section{Rim Effect}

In all included aneurysms the occurrence of a rim effect and its properties were evaluated. Such a hyperintense rim was noted in 23 aneurysms (96\%), whereas 1 aneurysm did not show a rim effect (4\%). Therefore, this case was excluded from further evaluation of the rim effect.

In the aneurysms that showed such a rim effect, a thin rim was observed in 17 cases (74\%) whereas a medium rim could be detected in 6 aneurysms (26\%). Regarding the completeness of this rim effect, a complete ring without any missing segments was observed in 1 case (4\%). In contrast, missing segments in the observed rim effect were noted in the remaining 22 aneurysms (96\%). Small blood vessels leaving the aneurysms or the parent vessels in close proximity to the rim effect were observed in 4 cases (18\%). A detailed list of these characteristics is given in Table 3.

According to these imaging parameters, further analyses were performed in the 22 aneurysms with an identifiable rim effect and missing segments within the rim effect. An example of this rim effect is shown in Fig. 1.

\section{CFD Analyses}

In these 22 included aneurysms, formation of 3D sur- 


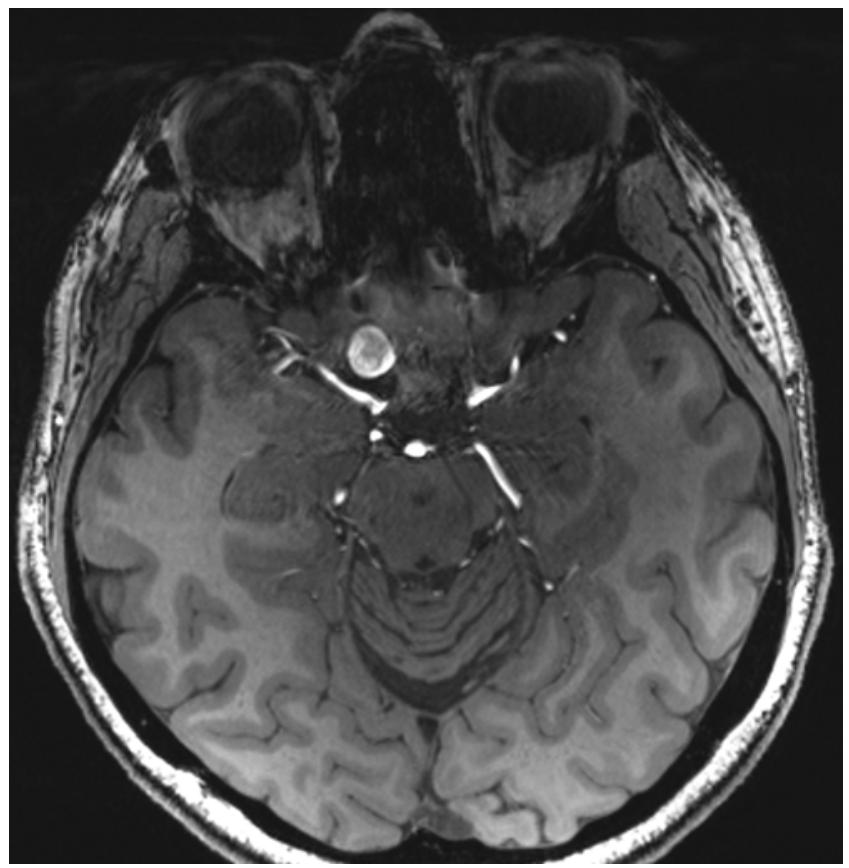

FIG. 1. Nonenhanced T1-weighted 7-T ultra-high field MR image of an ICA sidewall aneurysm showing a hyperintense intraluminal signal with an even brighter rim signal along the vessel wall, termed "rim effect."

face models and consequently CFD analyses were feasible in all cases. Each model was constructed by an average number of 7370 trigonal elements and further processed in COMSOL Multiphysics. For each parameter, values were calculated for the whole 3D model, however, comparison of the mean values was performed at the chosen locations. For an example of the rim effect compared to the performed CFD analyses, see Fig. 2.

In this comparison of all included aneurysms, significant differences were found for the mean wall shear stress (nonrim area $5.2 \pm 7.1 \mathrm{~Pa}$ vs rim area $1.9 \pm 1.4 \mathrm{~Pa}, \mathrm{p}=$ 0.043 ) and the vorticity (nonrim area $1052.4 \pm 1432.1 / \mathrm{sec}$ vs rim area $385.8 \pm 287.1 / \mathrm{sec}, \mathrm{p}=0.043$ ). Regarding the wall-directed force, no significant differences could be detected. Details of these comparisons are shown in Table 4.

Furthermore, the calculated values were also compared based on the position of the aneurysm in relation to the parent artery (vessel bifurcation or sidewall). In this sense, significantly higher values of the mean wall shear stress (nonrim area $4.3 \pm 4.5 \mathrm{~Pa}$ vs rim area $1.6 \pm 1.2 \mathrm{~Pa}, \mathrm{p}=$ 0.027 ) and the vorticity (nonrim area $863.9 \pm 911.5 / \mathrm{sec}$ vs rim area $327.7 \pm 252.3 / \mathrm{sec}, \mathrm{p}=0.027$ ) were also observed in the nonrim areas of aneurysms at vessel bifurcations. For the wall-directed force, no such significant difference could be detected. In contrast, in sidewall aneurysms no significant differences could be detected in any of these variables. A complete list of these values is provided in Table 4.

\section{Discussion}

IAs occur with a frequency of approximately $3 \%$ in the general population. ${ }^{35}$ However, because a significantly
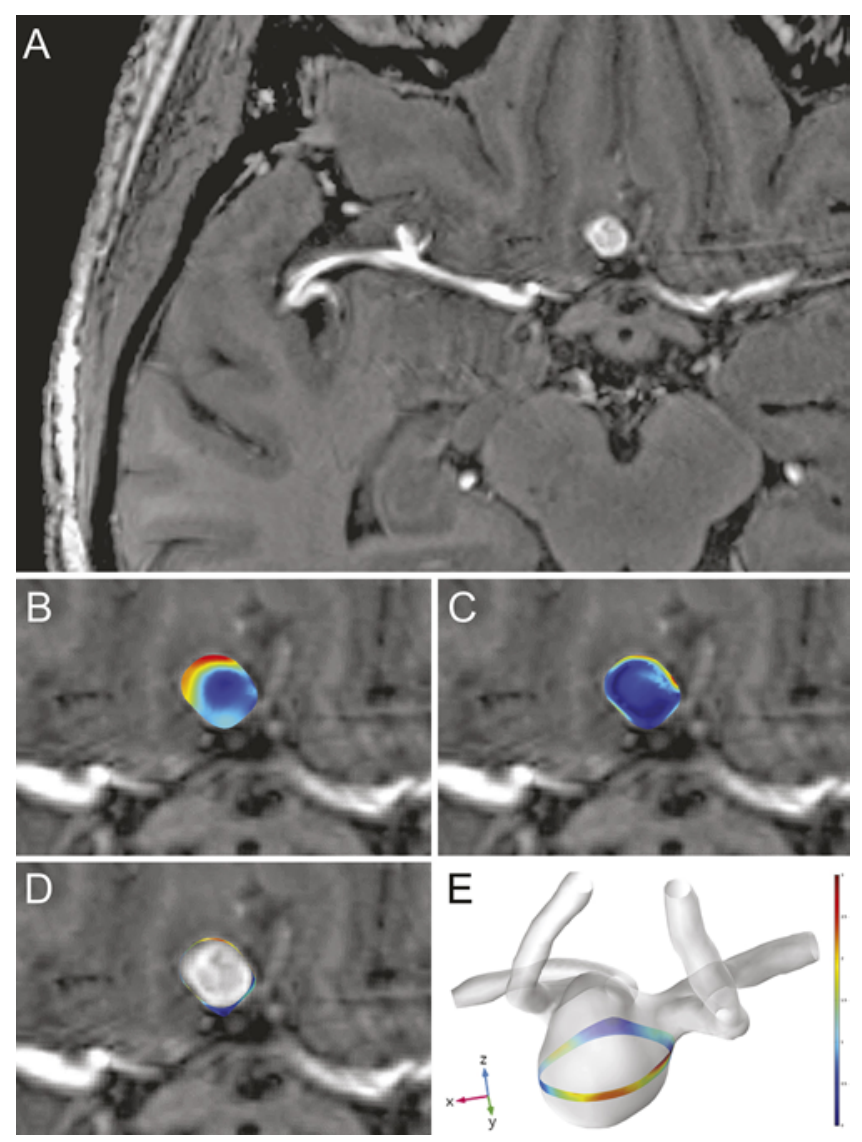

FIG. 2. A: Nonenhanced T1-weighted image of an anterior communicating artery aneurysm demonstrating focal disruption in the rim effect in the upper right quadrant. B-D: Addition of CFD analyses of the walldirected force $(B)$, vorticity $(C)$, and wall shear stress $(D)$ to the morphological MR image. E: Three-dimensional surface model with depiction of the evaluated image slice and the calculated wall shear stress.

lower number of patients suffer from SAH per year, only a small proportion of all aneurysms tend to rupture. ${ }^{9}$ Hence, attempts have been made to investigate factors leading to a higher risk of aneurysm rupture to select patients for treatment decisions. In this sense, a larger diameter, a history of hypertension or smoking, the aneurysm morphology, and the exact location of an IA account for the most reported risk factors. ${ }^{7,931,37}$ Nevertheless, clinical routine and further studies showed that most discovered aneurysms are small, and also such small aneurysms present with SAH. ${ }^{12,28}$ Therefore, there is a strong need to further identify patient-specific characteristics associated with an increased risk of aneurysm rupture.

With the advent of computer-based flow simulations, intraluminal blood flow parameters using CFD analyses were studied, because most IAs occur at locations prone to flow alterations such as vessel bifurcations. ${ }^{2,27}$ In this sense, changes in a number of different factors were investigated, with alterations in the wall shear stress being the most commonly mentioned. ${ }^{2,3,5,19,25}$ However, there is still an ongoing debate on whether a higher or lower wall shear stress is responsible for aneurysm development, growth, or rupture..$^{25,27}$ 
TABLE 4. Comparison of CFD parameters

\begin{tabular}{cccc}
\hline $\begin{array}{c}\text { CFD } \\
\text { Parameter }\end{array}$ & $\begin{array}{c}\text { Nonrim } \\
\text { Area }\end{array}$ & $\begin{array}{c}\text { Rim } \\
\text { Area }\end{array}$ & $\begin{array}{c}\text { p } \\
\text { Value }\end{array}$ \\
\hline Overall & & & \\
\hline Wall shear stress, Pa & $5.2(7.1)$ & $1.9(1.4)$ & 0.043 \\
\hline Vorticity, 1/sec & $1052.4(1432.1)$ & $385.8(287.1)$ & 0.043 \\
\hline Wall-directed force, Pa & $1101.5(1035.6)$ & $1111.1(1028.4)$ & 0.985 \\
\hline Aneurysm position & & & \\
\hline Vessel bifurcation & & & \\
\hline Wall shear stress, Pa & $4.3(4.5)$ & $1.6(1.2)$ & 0.027 \\
\hline Vorticity, 1/sec & $863.9(911.5)$ & $327.7(252.3)$ & 0.027 \\
\hline Wall-directed force, & $1088.7(1012.0)$ & $1084.8(996.2)$ & 0.991 \\
Pa & & & \\
\hline Sidewall & & & \\
\hline Wall shear stress, Pa & $9.4(14.3)$ & $3.2(1.6)$ & 0.454 \\
\hline Vorticity, 1/sec & $1900.8(2911.1)$ & $647.2(324.1)$ & 0.454 \\
\hline Wall-directed force, & $1179.0(1301.7)$ & $1229.4(1325.5)$ & 0.959 \\
Pa & & & \\
\hline
\end{tabular}

Data given as mean (SD) unless otherwise indicated. Boldface type indicates statistical significance.

Otherwise, investigations were performed on the importance of the aneurysm wall for the risk of rupture and subsequent hemorrhage..$^{3,8}$ Again, several changes within the vessel wall could be observed in both experimental studies and histological studies of clipped and resected aneurysm domes. ${ }^{8,15,17,18}$ However, both of these approaches require computer-based algorithms or resection of the dome to investigate the vessel wall after treatment.

In the last decade, attempts were made to study the intracranial vessel wall and associated pathologies by noninvasive neuroradiological imaging because changes within the vessel wall were observed in larger vessels in other parts of the human body using improved MRI. ${ }^{1,23,29,30}$ The first investigations on intracranial vessels evaluated a variety of intracranial vessel wall pathologies including atherosclerosis, inflammatory changes, and vessel wall dissection using both nonenhanced and contrast-enhanced MRI sequences. ${ }^{16,30}$ Some of these studies also tried to delineate the vessel wall in IAs. ${ }^{16,23,24}$ However, due to the low magnetic field strength used and the voxel size similar to the reported vessel wall thickness, these studies were critically reviewed..$^{29}$

To overcome this limitation, a small number of studies showed the value of 7-T MRI for depiction of IAs and delineation of the intracranial vessel wall in healthy volunteers and in a number of patients with different intracranial vascular pathologies, including IAs. ${ }^{11,33,34,39} \mathrm{Be}-$ sides delineation of the vessel wall directly, an interesting observation was made on 7-T MRI in intracranial vessels. In addition to this hyperintense intraluminal signal of the intracranial vasculature, the presence of a "rim enhancement" adjacent to the inner wall of IAs was noted on nonenhanced T1-weighted images. ${ }^{14,32,41}$ The impact of this finding, however, was not analyzed to date. Thus, the aim of this study was to compare this brighter hyperintense signal along the intracranial vessel wall in patients with unruptured IAs, with changes in intraluminal blood flow parameters.

\section{Present Study}

A total of 30 patients with 36 unruptured IAs were included, however, due to image distortion (especially close to the skull base) only 27 unruptured IAs showed appropriate image quality for further investigations. Of these 27 aneurysms, 3 were found to be fusiform aneurysms and thus were also excluded.

In addition to the hyperintense intraluminal signal on nonenhanced T1-weighted images described in the literature, an even brighter signal along the vessel wall was observed in 23 of 24 included aneurysms (96\%).14,32,38,41 This brighter signal was termed the "rim effect" and was further analyzed. Interestingly, focal irregularities could be depicted in 22 of these cases (96\%) with such a rim effect. CFD analyses were then performed, and the resulting values compared between specific locations, depending on the finding of a bright, clearly visible rim effect or if such focal irregularities could be depicted within this rim.

All CFD analyses were performed on 3D surface models of each aneurysm and the investigated parameters included wall shear stress, vorticity, and wall-directed force. In this evaluation, differences were observed for wall shear stress and vorticity with statistically significant higher values in the area with a missing rim effect $(5.2 \pm 7.1$ vs 1.9 $\pm 1.4 \mathrm{~Pa}, \mathrm{p}=0.043$, and $1052.4 \pm 1432.1 / \mathrm{sec}$ vs $385.8 \pm$ $287.1 / \mathrm{sec}, \mathrm{p}=0.043$, respectively). In a further subdivision of the different aneurysms, this effect remained statistically significant for bifurcation aneurysms $(4.3 \pm 4.5$ vs $1.6 \pm 1.2 \mathrm{~Pa}, \mathrm{p}=0.027$, and $863.9 \pm 911.5 / \mathrm{sec}$ vs $327.7 \pm$ $252.3 / \mathrm{sec}, \mathrm{p}=0.027$, respectively) but not for sidewall aneurysms. However, because only 4 aneurysms were located as sidewall aneurysms, this aspect needs further investigation with a larger number of patients. To the best of our knowledge, this study is the first to report and analyze this even brighter rim effect along the suspected vessel wall. Additionally, this is also the first description of such focal irregularities and interruptions in this rim effect.

In the current literature, there is an ongoing discussion about which alterations of blood flow are related to aneurysm growth and potential rupture. ${ }^{19}$ In this sense, aneurysms tend to occur in regions of typically high wall shear stress such as bifurcations as flow impinges on the aneurysm wall within the dome..$^{19,27}$ Also, vascular changes within the wall and bleb formation occurred in areas of high wall shear stress and a high wall shear stress gradient. ${ }^{5,27}$ In another analysis, higher wall shear stress values were found in ruptured aneurysms compared to unruptured IAs as well. ${ }^{4}$ However, growth of IAs has also been reported in both instances, at lower and higher wall shear stress values. ${ }^{27}$ Furthermore, various authors also reported morphological changes within the aneurysm wall, together with aneurysm rupture in cases with both lower and higher wall shear stress values..$^{25,27}$

Therefore, if these results are associated with aneurysm development, growth or rupture needs evaluation in further studies. However, these findings indicate that the described focal irregularities represent areas of alterations 
in blood flow within an IA as measured by CFD analyses from MPRAGE MRI at $7 \mathrm{~T}$.

\section{Limitations of the Study}

One limitation of this study is the evaluation of an indirect parameter of irregularities in the vessel wall of IAs instead of delineation of the vessel wall itself. However, depiction of the vessel wall itself with appropriate resolution requires long examination times not feasible in clinical routine MRI in patients harboring IAs. ${ }^{11}$ Additionally, the evaluation of wall irregularities by noninvasive neuroimaging and correlation to CFD analyses represents a computer-based evaluation, and a correlation with histological specimens from the aneurysm dome was planned. However, resection of the aneurysm dome was possible only in 1 patient and therefore a statistical analysis was not possible.

Furthermore, only aneurysms with a diameter of at least $5 \mathrm{~mm}$ were included in this study, and a large proportion of aneurysms display a diameter of less than $5 \mathrm{~mm}$ and still can present with rupture. However, this cutoff was chosen to analyze this finding in larger aneurysms in a first step. Therefore, the importance of this rim effect in smaller aneurysms needs to be evaluated in further studies.

\section{Conclusions}

The results of this study showed a bright hyperintense rim effect along the vessel wall on nonenhanced T1-weighted 7-T ultra-high field MR images. Within this rim effect, focal irregularities were noted in most cases. A comparison of these irregularities with CFD analyses showed higher values of the mean wall shear stress and vorticity in areas in which such a rim effect was missing. Therefore, these findings indicate alterations of the blood flow in these areas within an IA.

\section{Acknowledgments}

We would like to thank Ms. Ingrid Dobsak for assistance with the figures. This project has been funded by the Anniversary Fund for the promotion of Scientific Research and Teaching of the Austrian National Bank (OENB, project no. 15119) and was conducted as part of a $\mathrm{PhD}$ project of the first author.

\section{References}

1. Antiga L, Wasserman BA, Steinman DA: On the overestimation of early wall thickening at the carotid bulb by black blood MRI, with implications for coronary and vulnerable plaque imaging. Magn Reson Med 60:1020-1028, 2008

2. Castro M, Putman C, Radaelli A, Frangi A, Cebral J: Hemodynamics and rupture of terminal cerebral aneurysms. Acad Radiol 16:1201-1207, 2009

3. Cebral J, Ollikainen E, Chung BJ, Mut F, Sippola V, Jahromi $\mathrm{BR}$, et al: Flow conditions in the intracranial aneurysm lumen are associated with inflammation and degenerative changes of the aneurysm wall. AJNR Am J Neuroradiol 38:119-126, 2017

4. Cebral JR, Mut F, Weir J, Putman C: Quantitative characterization of the hemodynamic environment in ruptured and unruptured brain aneurysms. AJNR Am J Neuroradiol 32:145-151, 2011

5. Cebral JR, Sheridan M, Putman CM: Hemodynamics and bleb formation in intracranial aneurysms. AJNR Am J Neuroradiol 31:304-310, 2010
6. Cignoni P, Callieri M, Corsini M, Dellepiane M, Ganovelli F, Ranzuglia G: MeshLab: an Open-Source Mesh Processing Tool. Sixth Eurographics Italian Chapter Conference, 2008 (http://dx.doi.org/10.2312/LocalChapterEvents/ItalChap/ ItalianChapConf2008/129-136) [Accessed October 2, 2019]

7. Etminan N, Brown RD Jr, Beseoglu K, Juvela S, Raymond J, Morita A, et al: The unruptured intracranial aneurysm treatment score: a multidisciplinary consensus. Neurology 85:881-889, 2015

8. Frösen J, Piippo A, Paetau A, Kangasniemi M, Niemelä M, Hernesniemi J, et al: Remodeling of saccular cerebral artery aneurysm wall is associated with rupture: histological analysis of 24 unruptured and 42 ruptured cases. Stroke 35:22872293,2004

9. International Study of Unruptured Intracranial Aneurysms Investigators: Unruptured intracranial aneurysms - risk of rupture and risks of surgical intervention. $\mathbf{N}$ Engl J Med 339:1725-1733, 1998

10. Ishibashi T, Murayama Y, Urashima M, Saguchi T, Ebara M, Arakawa H, et al: Unruptured intracranial aneurysms: incidence of rupture and risk factors. Stroke 40:313-316, 2009

11. Kleinloog R, Korkmaz E, Zwanenburg JJM, Kuijf HJ, Visser F, Blankena R, et al: Visualization of the aneurysm wall: a 7.0-tesla magnetic resonance imaging study. Neurosurgery 75:614-622, 2014

12. Lee GJ, Eom KS, Lee C, Kim DW, Kang SD: Rupture of very small intracranial aneurysms: incidence and clinical characteristics. J Cerebrovasc Endovasc Neurosurg 17:217-222, 2015

13. Mach G, Heinzl R, Schwaha P, Stimpfl F, Weinbub J, Selberherr S, et al: A modular tool chain for high performance CFD simulations in intracranial aneurysms. AIP Conf Proc 1281:1647-1650, 2010

14. Maderwald S, Ladd SC, Gizewski ER, Kraff O, Theysohn JM, Wicklow K, et al: To TOF or not to TOF: strategies for non-contrast-enhanced intracranial MRA at 7 T. MAGMA 21:159-167, 2008

15. Marbacher S, Marjamaa J, Bradacova K, von Gunten M, Honkanen P, Abo-Ramadan U, et al: Loss of mural cells leads to wall degeneration, aneurysm growth, and eventual rupture in a rat aneurysm model. Stroke 45:248-254, 2014

16. Matouk CC, Mandell DM, Günel M, Bulsara KR, Malhotra A, Hebert R, et al: Vessel wall magnetic resonance imaging identifies the site of rupture in patients with multiple intracranial aneurysms: proof of principle. Neurosurgery 72:492-496, 2013

17. Meng H, Swartz DD, Wang Z, Hoi Y, Kolega J, Metaxa EM, et al: A model system for mapping vascular responses to complex hemodynamics at arterial bifurcations in vivo. Neurosurgery 59:1094-1101, 2006

18. Meng H, Wang Z, Hoi Y, Gao L, Metaxa E, Swartz DD, et al: Complex hemodynamics at the apex of an arterial bifurcation induces vascular remodeling resembling cerebral aneurysm initiation. Stroke 38:1924-1931, 2007

19. Munarriz PM, Gómez PA, Paredes I, Castaño-Leon AM, Cepeda S, Lagares A: Basic principles of hemodynamics and cerebral aneurysms. World Neurosurg 88:311-319, 2016

20. Nakatani H, Hashimoto N, Kang Y, Yamazoe N, Kikuchi $\mathrm{H}$, Yamaguchi S, et al: Cerebral blood flow patterns at major vessel bifurcations and aneurysms in rats. J Neurosurg 74:258-262, 1991

21. Newell DW, Aaslid R: Transcranial Doppler: clinical and experimental uses. Cerebrovasc Brain Metab Rev 4:122-143, 1992

22. Ohshima T, Miyachi S, Hattori K, Takahashi I, Ishii K, Izumi T, et al: Risk of aneurysmal rupture: the importance of neck orifice positioning-assessment using computational flow simulation. Neurosurgery 62:767-775, 2008

23. Park JK, Lee CS, Sim KB, Huh JS, Park JC: Imaging of the 
walls of saccular cerebral aneurysms with double inversion recovery black-blood sequence. J Magn Reson Imaging 30:1179-1183, 2009

24. Qiao Y, Steinman DA, Qin Q, Etesami M, Schär M, Astor $\mathrm{BC}$, et al: Intracranial arterial wall imaging using threedimensional high isotropic resolution black blood MRI at 3.0 Tesla. J Magn Reson Imaging 34:22-30, 2011

25. Qiu T, Jin G, Xing H, Lu H: Association between hemodynamics, morphology, and rupture risk of intracranial aneurysms: a computational fluid modeling study. Neurol Sci 38:1009-1018, 2017

26. Schöberl J: NETGEN An advancing front 2D/3D-mesh generator based on abstract rules. Comput Visual Sci 1:41-52, 1997

27. Sforza DM, Putman CM, Cebral JR: Computational fluid dynamics in brain aneurysms. Int J Numer Methods Biomed Eng 28:801-808, 2012

28. Sonobe M, Yamazaki T, Yonekura M, Kikuchi H: Small unruptured intracranial aneurysm verification study: SUAVe study, Japan. Stroke 41:1969-1977, 2010

29. Steinman DA, Antiga L, Wasserman BA: Overestimation of cerebral aneurysm wall thickness by black blood MRI? J Magn Reson Imaging 31:766, 2010

30. Swartz RH, Bhuta SS, Farb RI, Agid R, Willinsky RA, Terbrugge $\mathrm{KG}$, et al: Intracranial arterial wall imaging using high-resolution 3-tesla contrast-enhanced MRI. Neurology 72:627-634, 2009

31. Morita A, Kirino T, Hashi K, Aoki N, Fukuhara S, Hashimoto $\mathrm{N}$, et al: The natural course of unruptured cerebral aneurysms in a Japanese cohort. N Engl J Med 366:2474-2482, 2012

32. Umutlu L, Theysohn N, Maderwald S, Johst S, Lauenstein TC, Moenninghoff $\mathrm{C}$, et al: 7 Tesla MPRAGE imaging of the intracranial arterial vasculature: nonenhanced versus contrast-enhanced. Acad Radiol 20:628-634, 2013

33. van der Kolk AG, Hendrikse J, Brundel M, Biessels GJ, Smit EJ, Visser F, et al: Multi-sequence whole-brain intracranial vessel wall imaging at 7.0 tesla. Eur Radiol 23:2996-3004, 2013

34. van der Kolk AG, Zwanenburg JJ, Brundel M, Biessels GJ, Visser F, Luijten PR, et al: Intracranial vessel wall imaging at 7.0-T MRI. Stroke 42:2478-2484, 2011

35. Vlak MH, Algra A, Brandenburg R, Rinkel GJ: Prevalence of unruptured intracranial aneurysms, with emphasis on sex, age, comorbidity, country, and time period: a systematic review and meta-analysis. Lancet Neurol 10:626-636, 2011

36. Wermer MJH, van der Schaaf IC, Algra A, Rinkel GJE: Risk of rupture of unruptured intracranial aneurysms in relation to patient and aneurysm characteristics: an updated metaanalysis. Stroke 38:1404-1410, 2007
37. Wiebers DO, Whisnant JP, Huston J III, Meissner I, Brown RD Jr, Piepgras DG, et al: Unruptured intracranial aneurysms: natural history, clinical outcome, and risks of surgical and endovascular treatment. Lancet 362:103-110, 2003

38. Wrede KH, Dammann P, Mönninghoff C, Johst S, Maderwald S, Sandalcioglu IE, et al: Non-enhanced MR imaging of cerebral aneurysms: 7 Tesla versus 1.5 Tesla. PLoS One 9:e84562, 2014

39. Wrede KH, Matsushige T, Goericke SL, Chen B, Umutlu L, Quick $\mathrm{HH}$, et al: Non-enhanced magnetic resonance imaging of unruptured intracranial aneurysms at 7 Tesla: comparison with digital subtraction angiography. Eur Radiol 27:354364, 2017

40. Yushkevich PA, Piven J, Hazlett HC, Smith RG, Ho S, Gee JC, et al: User-guided 3D active contour segmentation of anatomical structures: significantly improved efficiency and reliability. Neuroimage 31:1116-1128, 2006

41. Zwanenburg JJM, Hendrikse J, Takahara T, Visser F, Luijten PR: MR angiography of the cerebral perforating arteries with magnetization prepared anatomical reference at $7 \mathrm{~T}$ : comparison with time-of-flight. J Magn Reson Imaging 28:1519-1526, 2008

\section{Disclosures}

Georg Mach, MSc, reports ownership in and being an employee of CVTec Cerebrovascular Technologies $\mathrm{GmbH}$ at the time the manuscript was written (but not currently).

\section{Author Contributions}

Conception and design: Trattnig, Millesi, Gruber. Acquisition of data: Millesi, Mach, Gruber. Analysis and interpretation of data: Trattnig, Millesi, Mach, Ricken, Gruber. Drafting the article: Millesi. Critically revising the article: all authors. Reviewed submitted version of manuscript: all authors. Approved the final version of the manuscript on behalf of all authors: Trattnig. Statistical analysis: Millesi, Mach, Gruber. Administrative/ technical/material support: all authors. Study supervision: Trattnig, Knosp, Hainfellner, Gruber.

\section{Correspondence}

Siegfried Trattnig: High Field MR Centre, Medical University of Vienna, Austria.siegfried.trattnig@meduniwien.ac.at. 